\title{
Left ventricular dysfunction during exercise in patients with angina pectoris and angiographically normal coronary arteries (syndrome $\mathrm{X}$ )
}

\author{
J. Taki ${ }^{1}$, K. Nakajima1, A. Muramori ${ }^{1}$, H. Yoshio², M. Shimizu², K. Hisada1
}

1 Department of Nuclear Medicine, Kanazawa University School of Medicine, 13-1 Takara-machi, Kanazawa, 920 Japan

2 The Second Department of Internal Medicine, Kanazawa University School of Medicine, 13-1 Takara-machi, Kanazawa, 920 Japan

Eur J Nucl Med (1994) 21:98-102

In Table 2 of the above article there were unfortunately some typesetting errors in the $P$ column. This table is therefore reprinted below in its entirety.

Table 2. Left ventricular function parameters in patients with syndrome $X$ and atypical chest pain (control)

\begin{tabular}{lccc}
\hline & Syndrome X & Control & $P$ \\
\hline Ejection fraction (\%) & & & \\
$\quad$ Rest & $62.1 \pm 6.7$ & $61.9 \pm 6.2$ & NS \\
At ST depression & $67.0 \pm 12.0$ & - & - \\
Peak exercise & $59.0 \pm 10.7$ & $76.6 \pm 8.9$ & $<0.001$ \\
EF-OS & $81.0 \pm 9.0$ & $88.0 \pm 9.0$ & $<0.05$ \\
$\Delta$ EF (rest-exercise) & $-3.1 \pm 9.5$ & $14.7 \pm 7.4$ & $<0.001$ \\
$\Delta$ EF (rest-OS) & $19.3 \pm 8.3$ & $26.4 \pm 7.3$ & $<0.001$ \\
Time to EF-OS (s) & $114 \pm 43$ & $74 \pm 43$ & $<0.05$ \\
End-diastolic volume (\%) & & & \\
At ST depression & $106 \pm 3.9$ & - & $\mathrm{NS}$ \\
Peak exercise & $107 \pm 4.8$ & $107 \pm 4.6$ & $\mathrm{NS}$ \\
EF-OS & $103 \pm 3.5$ & $102 \pm 4.3$ & \\
End-systolic volume (\%) & & & $<0.001$ \\
At ST depression & $89 \pm 27$ & - & $<0.05$ \\
Peak exercise & $116 \pm 28$ & $65 \pm 18$ & \\
EF-OS & $52 \pm 22$ & $31 \pm 19$ & \\
Cardiac output (\%) & & & \\
At ST depression & $192 \pm 53$ & - & \\
Peak exxercise & $207 \pm 61$ & $263 \pm 46$ & \\
EF-OS & $189 \pm 40$ & $219 \pm 40$ & \\
\hline
\end{tabular}

EF-OS, Haemodynamic parameters at ejection fraction overshoot; NS, non-significant 